\title{
Interlibrary Loan in New England
}

\begin{abstract}
A survey of characteristics of interlibrary loan (ILL) traffic in New England was conducted by the New England Library Information Network (NELINET) as part of research to develop a regional automated ILL system. Information was gathered on the patterns of lending shown by different types of libraries, the flow of ILL among the New England libraries, use of the OCLC data base for verification, age of materials requested via ILL, fill rates, and speed of turnaround. The data confirm that large research libraries loan more heavily than they borrow, but no clear trend is shown for ILL behavior of small to medium-sized libraries. A large percentage of lending is among libraries of the same type and within state borders. The survey is part of a project that attempts to develop a strategy for load-leveling within the ILL system.
\end{abstract}

I N November 1975 the Executive Committee of the New England Library Information Network (NELINET) mandated the development of a regional interlibrary communications system, with automated interlibrary loan (ILL) as the first subsystem. A Committee on Interlibrary Communication (CILC) was formed to advise the NELINET staff in the design and evaluation of such a system.

With the support of a grant from the United States Office of Education-Library Research and Demonstration Program, preliminary research began in September 1976. A review of the literature on automated ILL was one aim of this research; another was an ILL base-line statistical survey to obtain information about current ILL traffic patterns in New England. The survey was conducted in November and December 1976.

\section{PURPOSE AND METHOD}

Data gathered during the survey were intended for three purposes:

1. As a base against which to measure the impact of the automated ILL system when operational.

Libby Trudell is research analyst and James Wolper is systems consultant, New England Library Information Network, New England Board of Higher Education, Wellesley, Massachusetts.
2. For use in the design of a mathematical model to test techniques for loadleveling of ILL requests. One of the primary goals of this research was to investigate the potential for promoting equalization of the lending burden among libraries participating in an automated ILL system.

3. For use in the evaluation of a number of pertinent questions about ILL activity in New England.

The mathematical model of the ILL system has been described elsewhere in the library literature. ${ }^{1}$ This paper presents the results of the evaluation of the data, providing evidence to affirm certain premises about ILL activity. However, other widely held assumptions are called into question by the results. A complete report on this research is available through ERIC. ${ }^{2}$

The survey was designed by the CILC. Each institution participating in the survey recorded data for borrowing and lending transactions for the period of one month or thirty transactions, whichever came first. Those libraries exceeding thirty transactions in one month also recorded the total activity for each type of transaction. Data about turnaround time, fill rates, verification procedures, types of materials, and the flow of borrowing and lending among different types of libraries and different states were recorded.

The survey recipients included all NELINET members at the time of the sur- 
vey (sixty-two direct and forty-four indirect or consortium members). A nearly equal number of nonmember libraries throughout the region comprised the rest of the sample; the number of nonmember recipients was distributed equally in each of the six states. Public libraries of all sizes were included in the sample, and libraries from private, state, and community colleges were represented. Although the size of the sample was small, this diversity makes it a meaningful representation of New England's interlibrary loan traffic.

Of the 191 surveys distributed, 113 usable responses were received. The geographic distribution of returns showed a heavy percentage of respondents from Connecticut and Massachusetts, influenced by the large number of NELINET libraries in these states. NELINET member libraries comprised 47 percent of the total respondents. The overall response rate was $\mathbf{5 8}$ percent. A follow-up to nonrespondents revealed that ten to fifteen surveys were apparently lost in the mail or misplaced at the libraries. Other reasons noted were lack of staff time and belief on the part of small libraries that their minimal activity was not of sufficient impact to be included.

The 113 respondents (see table 1 for a breakdown of the sizes and types of respondents) reported 19,400 ILL transactions for the month of the survey. Of these, 11,500 were lending transactions, and 8,000 were borrowing transactions. This is a more balanced ratio than the two-to-one ratio of lending to borrowing reported in the 1972 study, by Vernon Palmour and others, of academic library lending. ${ }^{3}$ The difference is possibly caused by the more generalized nature of the sample population in the present study.

\section{Evaluation of Data}

The results of the survey have been quantified and evaluated in order to address the following characteristics of library and ILL activity that relate to the development and evaluation of a computerized ILL system:

\section{Size of Library}

It is a truism of ILL that large libraries receive an unequal share of ILL requests. This was borne out by the survey data, which showed that 29 percent of all lending in the survey period was done by five very large libraries, each with collections over $1,000,000$ volumes, which themselves accounted for only 11 percent of the borrowing. One of the libraries had a borrow/loan imbalance of one to five. These patterns are comparable to the lending patterns of very large academic libraries reported by $\mathrm{Pal}$ mour in the Westat study for the Association of Research Libraries. ${ }^{4}$.

It was expected that the data would show a direct relationship between size and ILL activity for all libraries, with borrowing leveling off and lending increasing with library size. In the sample as a whole there were fifty-four net borrowers and fifty-nine net lenders. Of the nineteen large libraries, only four were net borrowers, indicating the expected correlation between size and activity. However, no clear correlation was shown for the eighty-five libraries under 300,000 (classed as small and medium in size in the sample). Fifty (or 59 percent) were net borrowers, and thirty-five (or 41 percent) were net lenders. The borrowers and lenders were distributed randomly rather than being grouped by size. Some very small libraries loaned as much as ten times more than they borrowed, a behavior

TABLE 1

Survey Sample by Library Type and Size

\begin{tabular}{|c|c|c|c|c|c|}
\hline \multirow[t]{2}{*}{ Type of Library } & \multicolumn{4}{|c|}{ Size of Library } & Percent \\
\hline & Large & Medium & Small & Total & $(\mathrm{N}=113)$ \\
\hline Academic & 22 & 37 & 12 & 71 & $63 \%$ \\
\hline Public & 6 & 16 & 10 & 32 & $28 \%$ \\
\hline Special & - & - & 10 & 10 & $9 \%$ \\
\hline All Libraries & 28 & 53 & 32 & 113 & $100 \%$ \\
\hline
\end{tabular}

Large $=300,000+$ volumes (including extra-lange libraries)

Medium $=50,000-300,000$ volumes

Small $=$ up to 50,000 volumes 
especially evident of the special libraries in the sample.

Thus the data show that library size can be correlated with a library's status as a net borrower or net lender if a library is larger than 300,000 volumes. However, the activity of small and medium-sized libraries is unpredictable and dependent upon other factors such as subject strengths, geographic location, availability of location information, etc.

Most small libraries, whether net borrowers or net lenders, had a very small volume of ILL traffic. In fact, there appears to be a direct correlation between size of library and amount of borrowing. Of libraries with holdings under 100,000 , only 16 percent borrowed more than forty items in one month, while 28 percent borrowed under ten items. Of libraries with holdings of 100,000 to $200,000,36$ percent of the libraries borrowed more than forty items, and only 20 percent borrowed less than ten items. The borrowing activity continues to increase correspondingly with size of the library. The conclusion can be drawn that large libraries, although lending heavily, also borrow heavily.

\section{Types of Libraries}

No conclusion can be drawn from NELINET data about the relative percentage of all ILL requests in the region that are generated by each type of library, as the different types of libraries were not proportionally represented in the survey. However, the survey data show conclusively that most ILL is among libraries of the same type. Public libraries transact 80 percent of their ILL with publics, and academic libraries transact 66 percent of their ILL with other academics. (In this survey two state library systems were included in public library statistics.) Special and academic librar- ies borrow at an almost perfectly even rate from each other.

The percentage of requests filled by academic libraries for other academic libraries is somewhat higher than that found by Palmour, whose study showed that only 60 percent of items loaned by academic libraries were to other academic libraries. ${ }^{5}$ Since public libraries were not included at all in Palmour's study, it is not possible to compare NELINET's findings about the activity between public libraries with earlier research.

Table 2 shows a definitive imbalance in the lending activity between academic and public libraries. Academic libraries loaned an average of 9.4 items per month to public libraries, while borrowing 5.2 items per month, for a nonreciprocity ratio of 1 to 1.8 . The table also shows a greater volume of lending by academic than by public libraries, at a ratio of 1 to 1.5. Since there are more large academic than public libraries in the sample (see table 1 above), it is possible that the volume imbalance is a factor of the size of the libraries rather than type.

It would be helpful to construct a chart that shows the percentage of items borrowed by large libraries from medium, small from large, medium from medium, and so forth, so that some determination could be made about the nature of ILL traffic between different sizes of libraries. Unfortunately, the survey data do not provide that information, so it can only be conjectured that the low activity the data show for small libraries indicates that the individual small library-public, academic, or special-places little drain on the ILL system. Especially evident of this is the fact that the special libraries, all small in size, had an almost exactly one-to-one borrow/ loan ratio. This point should be dealt with and will be included in the survey analysis

TABLE 2

INTERLIBRARY LOAN AMONG TYPES OF LIBRARIES

(Average Number OF ITEMS PER INSTrTUtion)

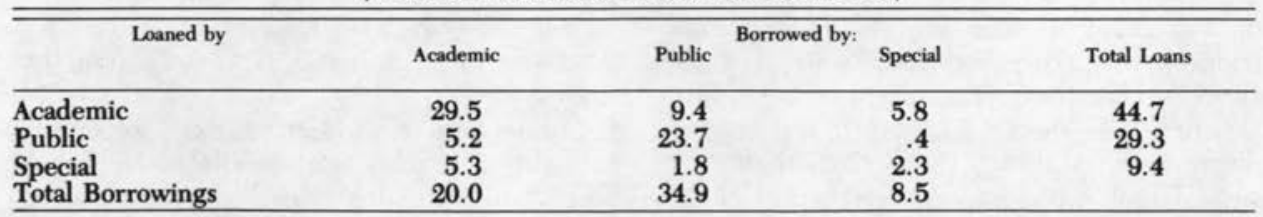


of the test made of the ILL system.

Some speculation can be made about the actual effect of the borrow/loan imbalance between public and academic libraries on the institutions. Items lent between public and academic libraries comprise 18 percent of all public activity and 21 percent of all academic activity. Thus each type of library individually is handling approximately onefifth of its total workload for the other type. This may indicate that academic libraries, because of their larger overall volume of lending, can absorb the demand made by public libraries. This theory is reinforced considering the average number of items loaned between public and academic libraries: The difference between the number loaned by academics and what they receive from publics is four items, less than 10 percent of all academic borrowing.

Thus the survey data confirm the premise that borrowing by public libraries is not reciprocal with the lending by public libraries to academic libraries. However, since the differential in the demand by public libraries represents only 10 percent of academic activity, it may be that this is a supportable imbalance within the system.

\section{Use of the OCLC Data Base for Verification}

On the average, libraries in the sample with access to a terminal (NELINET members only) verified 28 percent of all transactions on the OCLC data base. Most NELINET members used the OCLC system to verify their own borrowing requests more often than they received requests that had been so verified. However, this behavior is reversed for small academic libraries in the sample. While they verified 19 percent of their borrowing requests via OCLC, 29.5 percent of the loan requests they received had been verified by OCLC. It may be concluded that small academic libraries are attracting more ILL requests simply by the existence and visibility of their holdings symbol in the data base.

It should be noted that the survey results on this point are not fully reliable, as some participants expressed confusion over the term "verification." The intent of the survey was to determine OCLC system use for verification of location. Many participants assumed that bibliographic verification of the citation was intended. Despite this inconsistency, the results show a significant use of the data base for ILL activity.

\section{Age of Materials}

The data confirm a widely accepted view of the age of materials loaned through ILL. Forty percent of the requests recorded during the survey period were for materials published in the last three years. This is considerably more than the 21 percent found by Palmour's study within the same length of time. ${ }^{6}$ Only one request in ten was for an item published more than twenty years ago. After twenty years, the decline in the number of requests per year levels off, so that a request for an item published in 1945 is as likely as for an item from 1909.

It should also be noted that the high percentage of recent items indicates that most libraries are borrowing and loaning items that are in print, despite the ALA code that prohibits requests for such items. ${ }^{7}$

\section{In-State and Regional Interlibrary Loan}

It was expected that the survey data would reveal existing dependencies among the states, showing whether any one state is a heavy net borrower or net lender. Another aspect of this question was to determine the amount of ILL activity that goes on between New England and the other areas of the country. The data in table 3 show some interesting results on a stateby-state basis.

Almost all activity in New Hampshire is in-state (95 percent). This reflects a policy of the New Hampshire State Library not to route requests outside the state. As the number of requests that cannot be filled in-state is likely to be the same as in other states, which appear to fill between 20 to 30 percent of requests out-of-state, there may be a significant number of unfilled requests in New Hampshire.

Rhode Island's ILL traffic shows the highest ratio of out-of-state loans compared to borrowings: 32 to 24 percent. Rhode Island is the only New England state that is a significant net lender to other areas of the country.

Connecticut and Massachusetts resemble each other-each lends slightly more than it borrows but to such a small degree as to be 
TABLE 3

New England Interlibrary Loan Traffic Flow

\begin{tabular}{lcccccccc}
\hline \hline \multicolumn{1}{c}{ State } & \multicolumn{2}{c}{ In-State } & \multicolumn{2}{c}{$\begin{array}{c}\text { Out-of-State } \\
\text { (total) }\end{array}$} & \multicolumn{2}{c}{ Within New Eng. } & \multicolumn{2}{c}{ Out-of-State } \\
\hline & Loans & Borrowings & Loans & Borrowings & Loans & Borrowings & Loans & Borrowings \\
Conn. & $80.0 \%$ & $83.0 \%$ & $20.0 \%$ & $17.0 \%$ & $13.8 \%$ & $13.0 \%$ & $6.2 \%$ & $4.0 \%$ \\
Mass. & $79.0 \%$ & $80.5 \%$ & $21.0 \%$ & $19.5 \%$ & $9.4 \%$ & $8.1 \%$ & $11.6 \%$ & $11.4 \%$ \\
Maine & $77.0 \%$ & $74.0 \%$ & $23.0 \%$ & $26.0 \%$ & $10.4 \%$ & $11.5 \%$ & $12.6 \%$ & $4.5 \%$ \\
N.H. & $94.0 \%$ & $95.0 \%$ & $6.0 \%$ & $5.0 \%$ & $4.1 \%$ & $3.9 \%$ & $1.9 \%$ & $1.1 \%$ \\
R.I. & $68.0 \%$ & $76.0 \%$ & $32.0 \%$ & $24.0 \%$ & $16.4 \%$ & $14.9 \%$ & $15.6 \%$ & $9.1 \%$ \\
Vt. & $71.5 \%$ & $68.0 \%$ & $28.5 \%$ & $32.0 \%$ & $23.6 \%$ & $18.7 \%$ & $4.9 \%$ & $13.3 \%$ \\
\hline
\end{tabular}

almost insignificant. The amount that each state loans and borrows within its own boundaries is practically the same. Massachusetts has more interaction with libraries outside New England than does Connecticut.

Maine and Vermont show some similar behavior patterns, being more dependent on out-of-state sources for borrowing than the other states. Vermont depends on outside sources for 32 percent of its borrowing, while Maine depends on out-of-state for 26 percent of all borrowing. Vermont is the only state that is a consistent net borrower. Maine shows a relatively high percentage of loaning outside of New England. This may reflect the fact that the NELINET members in Maine include several large public libraries, which is not a general characteristic of NELINET membership. These libraries are very visible because of their access through the OCLC data base, and the higher percentage of public libraries in the other regional networks may cause a heavy use of Maine's public libraries.

The survey data indicate that 79 percent of all ILL transactions in New England are within state boundaries. This result is at some variance with the Palmour study, wherein only 64 percent of all requests are within the same state. ${ }^{8}$ It is likely that public libraries, which comprised almost 30 percent of the NELINET sample, are more likely to borrow in-state, especially through the medium of state library systems, than academic libraries. However, the percentage of ILL activity within the region was comparatively high in NELINET's study: 75-85 percent according to Palmour and 92 percent according to this study.

\section{Turnaround Times}

Time lapse was evaluated in terms of in- house processing time (from receipt of request to mailing item) and total turnaround time (from sending request to receiving item). Only the first-pass turnaround times were noted for most transactions. The data show that the average in-house turnaround time achieved by libraries in the survey was 2.5 days. More than 85 percent of all requests were answered within three days, and 32 percent were answered in less than a day. Only five libraries had an average in-house turnaround time longer than a week.

Although in-house turnaround time is fairly constant, total turnaround time-from initiation of request to receipt of the item at the borrowing library-varies significantly. Libraries received items borrowed in-state within an average of 8.5 days, whereas out-of-state transactions averaged as much as 26 days. The average overall turnaround time shown by the survey is 10.5 days. This is extremely fast in comparison to the turnaround times reported in a number of other studies, which averaged sixteen to eighteen days. ${ }^{9}$

\section{Effect of Volume on Turnaround Time}

The average turnaround time of the twenty-seven libraries that loaned more than 100 items in one month was 3.6 days, slightly higher than the $\mathbf{2 . 5}$ achieved overall. Only eight of these libraries had turnaround times longer than the average, but there was one with a turnaround time of fifteen days and several around seven days. It would appear that turnaround time does slow down in some highly impacted libraries; however, the library in the sample that did the most loaning ( 1,018 items) had a turnaround time of 2.3 days. This indicates 
that there may be other factors, such as size of ILL staff, which have an effect on delay.

\section{Fill Rate}

Success rate of the lending library in completing a request was called the "fill rate" in this survey. The overall fill rate for the requests made to the libraries in the survey was 93.6 percent. This percentage reflects only the final transactions for items that may have been referred several times; thus it is unrealistically high. In fact, the results are at a great variance with previous studies, which range from 71 percent filled in Palmour's study to 83 percent in other studies. ${ }^{10}$

The survey data show somewhat different fill rates for the different types of libraries. Academic libraries had the highest fill rate, with 96.5 percent of all requests made to these libraries being filled. Public libraries also showed a high fill rate, with 86.6 percent of all requests to them being filled. Special libraries drop to 79.9 percent filled; however, the low number of special libraries in the survey (only ten libraries) makes it difficult to justify drawing any conclusions about special library behavior in general.

The percentage of requests left unfilled tends to be higher in smaller libraries. This result is affected, however, by the number of requests each library receives. When a small library fills two out of three requests, its percentage of requests unfilled is 30 percent. Thus, the percentage is somewhat deceptive in characterizing fill rates.

\section{CONCLUSIONS}

The survey data indicate that New England libraries are successfully filling 93.6 percent of ILL requests, and they are filling them quickly. In-house ILL activity occurs within an average of 2.5 days, but actual time elapsed between initiating a request for a loan and receiving the item is considerably higher; it would seem that an automated system, such as that proposed by NELINET, could provide significant improvement of service in this respect.

The survey does not reveal the nature of the complex hierarchical systems that have been developed for public libraries by the New England states, but it does indicate that these systems are successful in filling ILL needs for their patrons. The survey data results about public library activity support the conclusion of Rolland Stevens that academic libraries are only a part of the total ILL picture. ${ }^{11} \mathrm{~A}$ survey of the activity experienced by all the state library networks in each of the New England states would be a valuable addition to the present study.

As expected, large libraries in the survey lent significantly more than they borrowed. However, the activity of small and medium-sized libraries varied to the extent that no generalization can be made about their ILL behavior. It can be assumed that many small libraries are probably underutilized for ILL because of lack of information about their holdings. A correlation can be drawn from the survey data between library size and volume of ILL activity: ILL activity, loaning and borrowing, increases steadily as size of the library increases.

Seventy-nine percent of transactions occurred within state boundaries, and 92.2 percent of all ILL traffic occurred within the New England region. A related behavior characteristic can be seen among the different types of libraries, as public libraries usually borrow from other public libraries and academics borrow mainly from academic libraries. Only special libraries show more interaction with other types of libraries than with other special libraries.

Results based on a limited survey cannot be considered a definitive portrait of New England ILL activity. However, it is a fair representation of the behavior of NELINET members and, as such, is certainly valuable in the design of an ILL system that initially will be primarily for that constituency.

As a sampling of many types of New England libraries, the survey provides a useful outline of ILL traffic within a region. Perhaps the most significant characteristic of this study was the inclusion of a range of different types of libraries. This range of participants provides data about the interaction among academic, public, and special libraries that was not available from the 1972 Palmour study and pressents a more balanced picture of interlibrary loan in a regional context. 


\section{REFERENCES}

1. James Wolper and Libby Trudell, "A Model of the NELINET Computerized ILL System: Testing Strategies for Load-Leveling," Journal of Library Automation 11:142-57 (June 1978).

2. "Demonstration and Evaluation of the Effects of Incentives on Resource Sharing Using a Computerized Interlibrary Communication System" (Wellesley, Mass.: New England Board of Higher Education, Dec. 1978 [ED 148 344]).

3. Vernon Palmour and others, A Study of the Characteristics, Costs, and Magnitude of Interlibrary Loans and Academic Libraries (Westport, Conn.: Greenwood Press, 1972), p.34.
4. Ibid., p.51.

5. Ibid., p. 4.

6. Ibid., p.39.

7. "National Interlibrary Loan Code, 1968," College \& Research Libraries News 29:27172 (Sept. 1968).

8. Palmour, A Study of the Characteristics, p. 35.

9. Rolland E. Stevens, "A Study of Interlibrary Loan," College \& Research Libraries 35:341 (Sept. 1974).

10. Palmour, A Study of the Characteristics, p.4; Stevens, "A Study of Interlibrary Loan," p. 341 .

11. Stevens, "A Study of Interlibrary Loan," p.336. 